\title{
Market Basket Analysis to Identify Stock Handling Patterns and Item Arrangement Patterns Using Apriori Algorithms
}

\author{
Sunardi*, Abdul Fadlil, Tresna Yudha Prawira \\ Masters Informatics Technology \\ Universitas Ahmad Dahlan \\ Yogyakarta \\ *sunardi@mti.uad.ac.id
}

\begin{abstract}
The process of managing the pattern of handling stock of goods and the pattern of arranging goods on store shelves requires an identification process by utilizing data from sales transaction results. Market basket analysis of sales transaction data using Apriori Algorithm stages produces an information in the form of association rules with a minimum support value of $50 \%$ and a minimum confidence of $60 \%$. It can be a reference in the arrangement of items on store shelves by referring to a combination of items that are often bought by consumers simultaneously. In addition, the stock inventory pattern can take advantage of the results of determining the high frequency value in the combination pattern 1 - itemset $\mathrm{C} 1$ with a minimum support value of $50 \%$ which is compared with the initial inventory.
\end{abstract}

Keywords: Apriori Algorithm, arrangement of goods, stock

\section{Introduction}

In the management of a minimarket, it is necessary to have a system management in the process of handling the patterns of stock handling and patterns of arrangement of goods, with the aim of structuring the goods to make it easier for customers to shop and patterns of handling stock of goods to provide the availability of goods needed by customers. Of the two, if not handled by an analysis of sales transaction data, it can be a problem for the development of minimarkets. Many business sellers of goods that assume that the sales transaction data report is only to be a report on several things, such as how many items are sold, how many items are still available, and how much profit is obtained. Sales transaction data can be used to help decisions in predicting the layout of goods so that consumers easily find the items sought and determine the prediction of the amount of stock in the future. These problems can be solved by market basket analysis using the stages of Apriori Algorithm, namely by identifying the value of support and confidence of goods sold at the minimarket. It can be a preference in the pattern of arrangement of goods based on customer habits in buying goods simultaneously and can also be a prediction of the stock of goods in the future [1].
Market basket analysis is the process of analyzing customer buying habits on each sales transaction data, by identifying associations between different items of the consumer shopping basket [2].

This research focuses on the event identifying the process of managing stock patterns of handling goods and structuring patterns of goods using Apriori Algorithm. This algorithm is used to find rules or measure the relationship between two or more items. Associative rules are expressed in terms of if they are antecedents, so they are consistent with the amount of support and confidence associated with the rules [3].

In a study entitled "Implementation of Apriori Algorithms with market basket analysis for product layout settings" explained that the association rules formed from the results of the discussion are used to regulate product placement in stores. Products that have high associations with other products will be placed close together, so as to facilitate consumers in buying products and store management in managing stock [4]. Maharani, et al. Also conduct research under the heading "Implementation of data mining for minimarket layout by applying association rule". The research aims to apply the association rule in the preparation of product layouts. From the rules generated, it can help companies in the preparation 
of product layouts [5]. Meanwhile, according to research conducted by Adyawangkara, et al with the title "Analysis of the rules of association between items in purchase transactions using data mining with Apriori Algorithm (case study: Gunungan minimarket, Central Java)", in that study aims to find the rules of association in the purchase of items in minimarkets to solve the problem of procurement of goods stock, determining promotional strategies, and arranging goods in minimarkets [6]. Research on the rules of association between items in identifying products that are sold and the relationship between products based on the conditions of the sales transaction data. [7] [8] [9] [10] [11] [12] [13] [14] [15].

\section{Research Methods}

This study conducted an experimental process with the aim of understanding the steps in the market basket analysis process using Apriori Algorithms in identifying patterns of handling stock items and patterns of arrangement of goods on store shelves. The stages in this study can be shown by Figure 1:

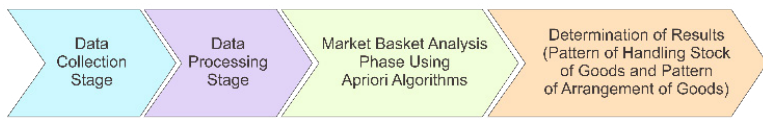

Figure 1. Research Process Stage

a. Data Collection Stage

The data used in this study were taken from the sales transaction database that is available at the Surya Mart minimarket. The mini market is a campus cooperative located at STMIK Muhammadiyah Paguyangan Brebes which is located on Jl. Pangeran Diponegoro, Kec. Paguyangan, Kab. Brebes. Postal Code 52276.

b. Data Processing Stage

At this stage the database scan process is carried out from the sales transaction table in order to calculate the number of purchases that occur on each item of goods. After that, the process is implemented into tabular data in the form of binary data with the statement 0 if the item was not purchased and 1 if the item was purchased on each item sales transaction identity.

c. Market Basket Analysis Phase Using Apriori Algorithms.

From the tabular data generated at the data processing stage, a market basket analysis process is performed using Apriori Algorithm to find high frequency values of sales transaction data by identifying support values and determining a minimum support value of $50 \%$. The next step is to establish a 2-item A association containing $\mathrm{B}$ by finding a confidence value, and determining a minimum confidence value of $60 \%$. Figure 2 shows the completion stage of the market basket analysis process using apriori algorithm:

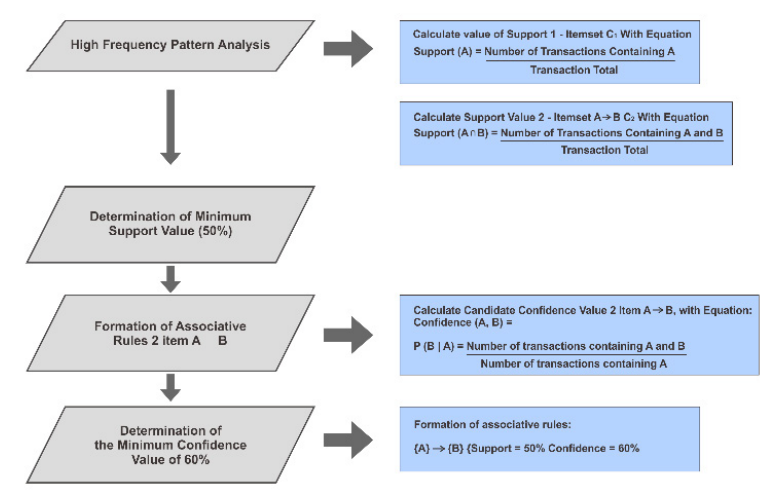

Figure 2. Market Basket Analysis Phase Using Apriori Algorithms.

d. Results Determination Stage

The final stage of this research is the process of determining the pattern of handling stock of goods and the arrangement of goods on store shelves from the results of the market basket analysis process using Apriori Algorithm in sales transaction data.

\section{Results snd Discussion}

\section{a. Data collection stage}

At this stage the data collection process is carried out from the database, in the form of sales data at the solar mart minimarket during the 1 (one) month transaction period as shown in Figure 3:

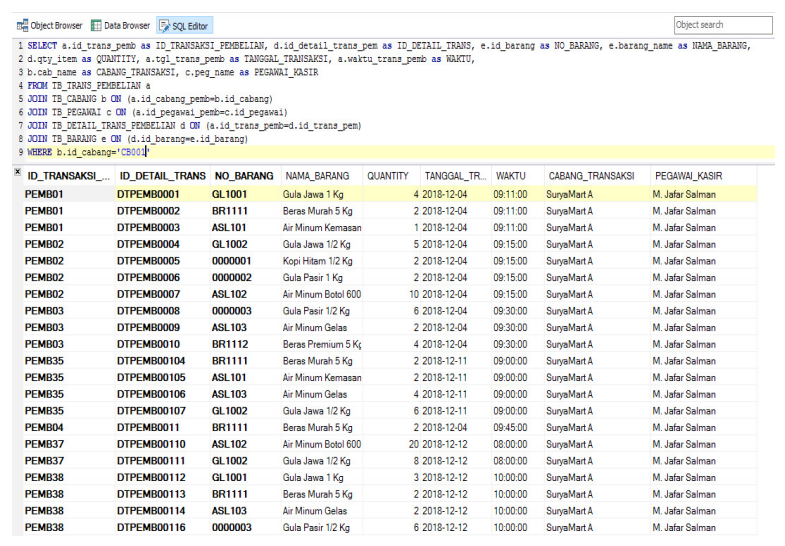

Figure 3. Data from the simulation of sales transactions.

After that, the data processing stage is carried out by scanning the database and changing it into tabular data.

\section{b. Data Processing Stage}

Data processing stage is done by scanning the database of sales transaction data, namely by identifying the number of items purchased at each transaction id, information $\mathrm{Y}$ and $\mathrm{N}$ on each item explains if "Y" means the item was purchased and if "N" means the item is not bought. Data is presented in tabular form as in table 1: 
Table 1. Number of items in each transaction id.

\begin{tabular}{|c|c|c|c|c|c|c|c|c|c|c|}
\hline $\begin{array}{l}\text { Transaction } \\
\text { ID }\end{array}$ & $\begin{array}{l}\text { Black } \\
\text { Coffee } \\
1 / 2 \mathrm{Kg}\end{array}$ & $\begin{array}{l}\text { Granulated } \\
\text { Sugar } 1 \mathrm{Kg}\end{array}$ & $\begin{array}{c}\text { Granulated } \\
\text { Sugar 1/2 } \\
\mathrm{Kg}\end{array}$ & $\begin{array}{l}\text { Gallon } \\
\text { Bottled } \\
\text { Water }\end{array}$ & $\begin{array}{l}600 \mathrm{ml} \\
\text { Bottled } \\
\text { Water }\end{array}$ & $\begin{array}{c}\text { Glass } \\
\text { drinking } \\
\text { water }\end{array}$ & $\begin{array}{l}\text { Cheap } \\
\text { rice } 5 \mathrm{~kg}\end{array}$ & $\begin{array}{l}\text { Premium } \\
\text { Rice } 5 \mathrm{Kg}\end{array}$ & $\begin{array}{c}\text { Javanese } \\
\text { Sugar } 1 \\
\text { Kg }\end{array}$ & $\begin{array}{c}\text { Java } \\
\text { Sugar } \\
1 / 2 \mathrm{Kg}\end{array}$ \\
\hline PEMB01 & $\bar{Y}$ & $\mathrm{Y}$ & $\bar{Y}$ & $\mathrm{Y}$ & $\mathrm{N}$ & $\mathrm{N}$ & $\mathrm{Y}$ & $\mathrm{N}$ & 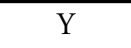 & $\mathrm{N}$ \\
\hline PEMB02 & $\mathrm{N}$ & $\mathrm{Y}$ & $\mathrm{N}$ & $\mathrm{N}$ & $\mathrm{Y}$ & $\mathrm{N}$ & $\mathrm{N}$ & $\mathrm{Y}$ & Y & $\mathrm{Y}$ \\
\hline PEMB03 & $\mathrm{N}$ & Y & $\mathrm{N}$ & $\mathrm{N}$ & $\mathrm{N}$ & $\mathrm{Y}$ & $\mathrm{N}$ & Y & Y & $\mathrm{N}$ \\
\hline PEMB04 & Y & Y & $\mathrm{Y}$ & $\mathrm{N}$ & $\mathrm{N}$ & $\mathrm{N}$ & $\mathrm{Y}$ & Y & $\mathrm{N}$ & $\mathrm{N}$ \\
\hline PEMB10 & $\mathrm{Y}$ & $\mathrm{N}$ & $\mathrm{N}$ & $\mathrm{Y}$ & $\mathrm{N}$ & $\mathrm{N}$ & $\mathrm{N}$ & $\mathrm{N}$ & $\mathrm{Y}$ & Y \\
\hline PEMB11 & Y & $\mathrm{N}$ & $\mathrm{Y}$ & $\mathrm{N}$ & $\mathrm{N}$ & $\mathrm{N}$ & $\mathrm{Y}$ & $\mathrm{Y}$ & $\mathrm{Y}$ & Y \\
\hline PEMB15 & $\mathrm{Y}$ & $\mathrm{N}$ & $\mathrm{Y}$ & $\mathrm{Y}$ & $\mathrm{N}$ & $\mathrm{N}$ & $\mathrm{Y}$ & $\mathrm{Y}$ & $\mathrm{Y}$ & Y \\
\hline PEMB16 & $\mathrm{N}$ & $\mathrm{Y}$ & $\mathrm{Y}$ & $\mathrm{Y}$ & $\mathrm{Y}$ & $\mathrm{Y}$ & Y & $\mathrm{N}$ & $\mathrm{N}$ & $\mathrm{N}$ \\
\hline PEMB19 & $\mathrm{N}$ & $\mathrm{Y}$ & $\mathrm{N}$ & $\mathrm{Y}$ & $\mathrm{Y}$ & $\mathrm{N}$ & $\mathrm{N}$ & $\mathrm{N}$ & $\mathrm{N}$ & $\mathrm{N}$ \\
\hline PEMB20 & Y & Y & $\mathrm{Y}$ & $\mathrm{N}$ & $\mathrm{N}$ & $\mathrm{N}$ & Y & Y & $\mathrm{Y}$ & $\mathrm{N}$ \\
\hline PEMB23 & $\mathrm{N}$ & $\mathrm{Y}$ & $\mathrm{Y}$ & $\mathrm{Y}$ & $\mathrm{N}$ & $\mathrm{N}$ & Y & $\mathrm{N}$ & $\mathrm{Y}$ & $\mathrm{N}$ \\
\hline PEMB24 & Y & $\mathrm{N}$ & $\mathrm{N}$ & $\mathrm{Y}$ & $\mathrm{Y}$ & $\mathrm{N}$ & Y & $\mathrm{Y}$ & $\mathrm{N}$ & Y \\
\hline PEMB27 & $\mathrm{N}$ & $\mathrm{Y}$ & Y & $\mathrm{N}$ & $\mathrm{N}$ & $\mathrm{N}$ & $\mathrm{Y}$ & $\mathrm{N}$ & $\mathrm{Y}$ & Y \\
\hline PEMB28 & Y & $\mathrm{N}$ & $\mathrm{N}$ & $\mathrm{N}$ & $\mathrm{N}$ & $\mathrm{N}$ & Y & $\mathrm{Y}$ & $\mathrm{Y}$ & $\mathrm{N}$ \\
\hline PEMB31 & $\mathrm{N}$ & $\mathrm{N}$ & $\mathrm{N}$ & $\mathrm{Y}$ & $\mathrm{N}$ & $\mathrm{N}$ & Y & Y & $\mathrm{Y}$ & $\mathrm{N}$ \\
\hline PEMB32 & Y & $\mathrm{Y}$ & $\mathrm{Y}$ & Y & $\mathrm{N}$ & $\mathrm{N}$ & Y & Y & $\mathrm{N}$ & $\mathrm{Y}$ \\
\hline PEMB35 & $\mathrm{N}$ & $\mathrm{N}$ & $\mathrm{Y}$ & $\mathrm{Y}$ & $\mathrm{N}$ & $\mathrm{Y}$ & $\mathrm{Y}$ & $\mathrm{N}$ & $\mathrm{Y}$ & Y \\
\hline PEMB37 & $\mathrm{Y}$ & $\mathrm{Y}$ & $\mathrm{Y}$ & $\mathrm{Y}$ & $\mathrm{Y}$ & $\mathrm{N}$ & $\mathrm{Y}$ & $\mathrm{N}$ & $\mathrm{N}$ & Y \\
\hline PEMB38 & $\mathrm{N}$ & $\mathrm{N}$ & $\mathrm{Y}$ & $\mathrm{N}$ & $\mathrm{N}$ & $\mathrm{Y}$ & $\mathrm{Y}$ & $\mathrm{N}$ & $\mathrm{Y}$ & $\mathrm{N}$ \\
\hline PEMB41 & Y & $\mathrm{Y}$ & $\mathrm{Y}$ & $\mathrm{Y}$ & $\mathrm{N}$ & $\mathrm{Y}$ & $\mathrm{N}$ & $\mathrm{N}$ & $\mathrm{Y}$ & $\mathrm{N}$ \\
\hline PEMB43 & Y & Y & Y & Y & $\mathrm{Y}$ & $\mathrm{N}$ & $\mathrm{N}$ & $\mathrm{N}$ & $\mathrm{N}$ & $\mathrm{N}$ \\
\hline PEMB44 & $\mathrm{Y}$ & Y & $\mathrm{N}$ & $\mathrm{N}$ & $\mathrm{N}$ & $\mathrm{N}$ & Y & $\mathrm{Y}$ & $\mathrm{N}$ & Y \\
\hline PEMB47 & Y & Y & $\mathrm{N}$ & $\mathrm{N}$ & $\mathrm{N}$ & $\mathrm{Y}$ & $\mathrm{N}$ & $\mathrm{Y}$ & $\mathrm{N}$ & $\mathrm{N}$ \\
\hline PEMB49 & $\mathrm{N}$ & $\mathrm{N}$ & $\mathrm{N}$ & $\mathrm{N}$ & $\mathrm{N}$ & $\mathrm{N}$ & $\mathrm{Y}$ & $\mathrm{Y}$ & $\mathrm{N}$ & $\mathrm{N}$ \\
\hline PEMB50 & $\mathrm{N}$ & $\mathrm{N}$ & $\mathrm{Y}$ & $\mathrm{Y}$ & $\mathrm{N}$ & $\mathrm{N}$ & $\mathrm{Y}$ & $\mathrm{N}$ & $\mathrm{Y}$ & $\mathrm{N}$ \\
\hline PEMB53 & Y & $\mathrm{Y}$ & $\mathrm{N}$ & $\mathrm{N}$ & $\mathrm{Y}$ & $\mathrm{N}$ & Y & $\mathrm{N}$ & $\mathrm{Y}$ & $\mathrm{N}$ \\
\hline PEMB55 & $\mathrm{N}$ & $\mathrm{N}$ & $\mathrm{N}$ & Y & $\mathrm{N}$ & $\mathrm{N}$ & $\mathrm{N}$ & Y & $\mathrm{N}$ & Y \\
\hline PEMB56 & $\mathrm{Y}$ & $\mathrm{Y}$ & $\mathrm{Y}$ & $\mathrm{N}$ & $\mathrm{N}$ & $\mathrm{Y}$ & $\mathrm{N}$ & $\mathrm{N}$ & $\mathrm{N}$ & $\mathrm{N}$ \\
\hline PEMB59 & $\mathrm{N}$ & $\mathrm{N}$ & $\mathrm{N}$ & $\mathrm{N}$ & $\mathrm{N}$ & $\mathrm{N}$ & $\mathrm{Y}$ & $\mathrm{Y}$ & $\mathrm{N}$ & $\mathrm{N}$ \\
\hline PEMB61 & $\mathrm{Y}$ & $\mathrm{Y}$ & $\mathrm{N}$ & $\mathrm{N}$ & $\mathrm{N}$ & $\mathrm{Y}$ & $\mathrm{N}$ & $\mathrm{Y}$ & $\mathrm{N}$ & $\mathrm{N}$ \\
\hline PEMB62 & $\mathrm{N}$ & $\mathrm{N}$ & $\mathrm{Y}$ & $\mathrm{N}$ & $\mathrm{Y}$ & $\mathrm{N}$ & $\mathrm{Y}$ & $\mathrm{N}$ & $\mathrm{N}$ & $\mathrm{N}$ \\
\hline PEMB65 & $\mathrm{N}$ & $\mathrm{N}$ & $\mathrm{N}$ & $\mathrm{Y}$ & $\mathrm{N}$ & $\mathrm{N}$ & $\mathrm{N}$ & $\mathrm{Y}$ & $\mathrm{Y}$ & $\mathrm{N}$ \\
\hline PEMB67 & $\mathrm{N}$ & $\mathrm{Y}$ & $\mathrm{N}$ & $\mathrm{N}$ & $\mathrm{N}$ & $\mathrm{N}$ & $\mathrm{Y}$ & $\mathrm{N}$ & $\mathrm{N}$ & $\mathrm{N}$ \\
\hline PEMB68 & $\mathrm{N}$ & $\mathrm{N}$ & $\mathrm{Y}$ & $\mathrm{Y}$ & $\mathrm{N}$ & $\mathrm{N}$ & $\mathrm{N}$ & $\mathrm{N}$ & $\mathrm{Y}$ & $\mathrm{N}$ \\
\hline PEMB71 & $\mathrm{N}$ & $\mathrm{Y}$ & $\mathrm{N}$ & $\mathrm{N}$ & $\mathrm{N}$ & $\mathrm{Y}$ & Y & $\mathrm{N}$ & $\mathrm{N}$ & $\mathrm{N}$ \\
\hline PEMB73 & $\mathrm{N}$ & $\mathrm{N}$ & $\mathrm{N}$ & $\mathrm{Y}$ & $\mathrm{N}$ & $\mathrm{N}$ & $\mathrm{Y}$ & Y & $\mathrm{Y}$ & $\mathrm{N}$ \\
\hline PEMB74 & $\mathrm{Y}$ & $\mathrm{Y}$ & $\mathrm{N}$ & $\mathrm{N}$ & $\mathrm{N}$ & $\mathrm{N}$ & $\mathrm{N}$ & $\mathrm{N}$ & $\mathrm{Y}$ & $\mathrm{N}$ \\
\hline PEMB77 & $\mathrm{N}$ & $\mathrm{N}$ & $\mathrm{Y}$ & $\mathrm{Y}$ & $\mathrm{N}$ & $\mathrm{N}$ & $\mathrm{N}$ & $\mathrm{Y}$ & $\mathrm{Y}$ & $\mathrm{N}$ \\
\hline PEMB79 & Y & $\mathrm{N}$ & $\mathrm{Y}$ & Y & $\mathrm{N}$ & $\mathrm{N}$ & Y & $\mathrm{N}$ & $\mathrm{N}$ & $\mathrm{Y}$ \\
\hline PEMB80 & $\mathrm{N}$ & Y & $\mathrm{N}$ & $\mathrm{N}$ & $\mathrm{N}$ & $\mathrm{Y}$ & $\mathrm{N}$ & $\mathrm{N}$ & $\mathrm{N}$ & $\mathrm{N}$ \\
\hline PEMB81 & Y & Y & $\mathrm{Y}$ & $\mathrm{Y}$ & $\mathrm{N}$ & $\mathrm{N}$ & $\mathrm{N}$ & $\mathrm{Y}$ & $\mathrm{Y}$ & $\mathrm{N}$ \\
\hline PEMB82 & $\mathrm{N}$ & $\mathrm{N}$ & $\mathrm{N}$ & $\mathrm{N}$ & $\mathrm{N}$ & $\mathrm{Y}$ & $\mathrm{Y}$ & $\mathrm{Y}$ & $\mathrm{N}$ & $\mathrm{N}$ \\
\hline PEMB88 & $\mathrm{Y}$ & $\mathrm{N}$ & $\mathrm{Y}$ & $\mathrm{Y}$ & $\mathrm{N}$ & $\mathrm{N}$ & $\mathrm{Y}$ & $\mathrm{N}$ & $\mathrm{Y}$ & $\mathrm{N}$ \\
\hline PEMB89 & $\mathrm{N}$ & $\mathrm{N}$ & $\mathrm{N}$ & $\mathrm{Y}$ & $\mathrm{N}$ & $\mathrm{Y}$ & $\mathrm{N}$ & $\mathrm{Y}$ & $\mathrm{N}$ & $\mathrm{N}$ \\
\hline PEMB91 & $\mathrm{Y}$ & $\mathrm{Y}$ & $\mathrm{Y}$ & $\mathrm{Y}$ & $\mathrm{N}$ & $\mathrm{N}$ & $\mathrm{N}$ & Y & $\mathrm{N}$ & $\mathrm{N}$ \\
\hline PEMB92 & $\mathrm{Y}$ & Y & Y & $\mathrm{Y}$ & $\mathrm{Y}$ & $\mathrm{N}$ & $\mathrm{N}$ & $\mathrm{Y}$ & $\mathrm{Y}$ & $\mathrm{N}$ \\
\hline PEMB95 & $\mathrm{N}$ & $\mathrm{N}$ & Y & $\mathrm{Y}$ & $\mathrm{N}$ & $\mathrm{N}$ & $\mathrm{N}$ & $\mathrm{N}$ & $\mathrm{Y}$ & $\mathrm{N}$ \\
\hline PEMB97 & $\mathrm{Y}$ & Y & Y & $\mathrm{Y}$ & $\mathrm{N}$ & $\mathrm{N}$ & $\mathrm{N}$ & Y & $\mathrm{Y}$ & $\mathrm{N}$ \\
\hline PEMB98 & $\mathrm{Y}$ & $\mathrm{Y}$ & Y & $\mathrm{Y}$ & $\mathrm{N}$ & $\mathrm{N}$ & $\mathrm{N}$ & Y & $\mathrm{Y}$ & $\mathrm{N}$ \\
\hline PEMB99 & $\mathrm{Y}$ & $\mathrm{N}$ & $\mathrm{Y}$ & $\mathrm{N}$ & $\mathrm{N}$ & $\mathrm{N}$ & $\mathrm{N}$ & $\mathrm{Y}$ & $\mathrm{N}$ & $\mathrm{N}$ \\
\hline
\end{tabular}


From the data in Table 1 which contains the items column $\quad \mathrm{Y}=1$ and $\mathrm{N}=0$ with the aim that it is easy to count each with information $\mathrm{Y}$ and $\mathrm{N}$, it is changed in binary form to item on each transaction, as shown in Table 2:

Table 2. The number of transaction items is changed in binary form.

\begin{tabular}{|c|c|c|c|c|c|c|c|c|c|c|}
\hline Transaction ID & $\begin{array}{l}\text { Black } \\
\text { Coffee } \\
1 / 2 \mathrm{Kg}\end{array}$ & $\begin{array}{l}\text { Granulated } \\
\text { Sugar } 1 \mathrm{Kg}\end{array}$ & $\begin{array}{c}\text { Granulated } \\
\text { Sugar 1/2 Kg }\end{array}$ & $\begin{array}{c}\text { Gallon } \\
\text { Bottled Water }\end{array}$ & $\begin{array}{l}600 \mathrm{ml} \\
\text { Bottled } \\
\text { Water }\end{array}$ & $\begin{array}{c}\text { Glass } \\
\text { drinking } \\
\text { water }\end{array}$ & $\begin{array}{l}\text { Cheap } \\
\text { rice } 5 \mathrm{~kg}\end{array}$ & $\begin{array}{l}\text { Premium } \\
\text { Rice } 5 \mathrm{Kg}\end{array}$ & $\begin{array}{c}\text { Javanese } \\
\text { Sugar } 1 \\
\text { Kg }\end{array}$ & $\begin{array}{c}\text { Java } \\
\text { Sugar 1/2 } \\
\text { Kg }\end{array}$ \\
\hline PEMB01 & 1 & 1 & 1 & 1 & 0 & 0 & 1 & 0 & 1 & 0 \\
\hline PEMB02 & 0 & 1 & 0 & 0 & 1 & 0 & 0 & 1 & 1 & 1 \\
\hline PEMB03 & 0 & 1 & 0 & 0 & 0 & 1 & 0 & 1 & 1 & 0 \\
\hline PEMB04 & 1 & 1 & 1 & 0 & 0 & 0 & 1 & 1 & 0 & 0 \\
\hline PEMB10 & 1 & 0 & 0 & 1 & 0 & 0 & 0 & 0 & 1 & 1 \\
\hline PEMB11 & 1 & 0 & 1 & 0 & 0 & 0 & 1 & 1 & 1 & 1 \\
\hline PEMB15 & 1 & 0 & 1 & 1 & 0 & 0 & 1 & 1 & 1 & 1 \\
\hline PEMB16 & 0 & 1 & 1 & 1 & 1 & 1 & 1 & 0 & 0 & 0 \\
\hline PEMB19 & 0 & 1 & 0 & 1 & 1 & 0 & 0 & 0 & 0 & 0 \\
\hline PEMB20 & 1 & 1 & 1 & 0 & 0 & 0 & 1 & 1 & 1 & 0 \\
\hline PEMB23 & 0 & 1 & 1 & 1 & 0 & 0 & 1 & 0 & 1 & 0 \\
\hline PEMB24 & 1 & 0 & 0 & 1 & 1 & 0 & 1 & 1 & 0 & 1 \\
\hline PEMB27 & 0 & 1 & 1 & 0 & 0 & 0 & 1 & 0 & 1 & 1 \\
\hline PEMB28 & 1 & 0 & 0 & 0 & 0 & 0 & 1 & 1 & 1 & 0 \\
\hline PEMB31 & 0 & 0 & 0 & 1 & 0 & 0 & 1 & 1 & 1 & 0 \\
\hline PEMB32 & 1 & 1 & 1 & 1 & 0 & 0 & 1 & 1 & 0 & 1 \\
\hline PEMB35 & 0 & 0 & 1 & 1 & 0 & 1 & 1 & 0 & 1 & 1 \\
\hline PEMB37 & 1 & 1 & 1 & 1 & 1 & 0 & 1 & 0 & 0 & 1 \\
\hline PEMB38 & 0 & 0 & 1 & 0 & 0 & 1 & 1 & 0 & 1 & 0 \\
\hline PEMB41 & 1 & 1 & 1 & 1 & 0 & 1 & 0 & 0 & 1 & 0 \\
\hline PEMB43 & 1 & 1 & 1 & 1 & 1 & 0 & 0 & 0 & 0 & 0 \\
\hline PEMB 44 & 1 & 1 & 0 & 0 & 0 & 0 & 1 & 1 & 0 & 1 \\
\hline PEMB47 & 1 & 1 & 0 & 0 & 0 & 1 & 0 & 1 & 0 & 0 \\
\hline PEMB49 & 0 & 0 & 0 & 0 & 0 & 0 & 1 & 1 & 0 & 0 \\
\hline PEMB50 & 0 & 0 & 1 & 1 & 0 & 0 & 1 & 0 & 1 & 0 \\
\hline PEMB53 & 1 & 1 & 0 & 0 & 1 & 0 & 1 & 0 & 1 & 0 \\
\hline PEMB55 & 0 & 0 & 0 & 1 & 0 & 0 & 0 & 1 & 0 & 1 \\
\hline PEMB56 & 1 & 1 & 1 & 0 & 0 & 1 & 0 & 0 & 0 & 0 \\
\hline PEMB59 & 0 & 0 & 0 & 0 & 0 & 0 & 1 & 1 & 0 & 0 \\
\hline PEMB61 & 1 & 1 & 0 & 0 & 0 & 1 & 0 & 1 & 0 & 0 \\
\hline PEMB62 & 0 & 0 & 1 & 0 & 1 & 0 & 1 & 0 & 0 & 0 \\
\hline PEMB65 & 0 & 0 & 0 & 1 & 0 & 0 & 0 & 1 & 1 & 0 \\
\hline PEMB67 & 0 & 1 & 0 & 0 & 0 & 0 & 1 & 0 & 0 & 0 \\
\hline PEMB68 & 0 & 0 & 1 & 1 & 0 & 0 & 0 & 0 & 1 & 0 \\
\hline PEMB71 & 0 & 1 & 0 & 0 & 0 & 1 & 1 & 0 & 0 & 0 \\
\hline PEMB73 & 0 & 0 & 0 & 1 & 0 & 0 & 1 & 1 & 1 & 0 \\
\hline PEMB74 & 1 & 1 & 0 & 0 & 0 & 0 & 0 & 0 & 1 & 0 \\
\hline PEMB77 & 0 & 0 & 1 & 1 & 0 & 0 & 0 & 1 & 1 & 0 \\
\hline PEMB79 & 1 & 0 & 1 & 1 & 0 & 0 & 1 & 0 & 0 & 1 \\
\hline PEMB80 & 0 & 1 & 0 & 0 & 0 & 1 & 0 & 0 & 0 & 0 \\
\hline PEMB81 & 1 & 1 & 1 & 1 & 0 & 0 & 0 & 1 & 1 & 0 \\
\hline PEMB82 & 0 & 0 & 0 & 0 & 0 & 1 & 1 & 1 & 0 & 0 \\
\hline PEMB88 & 1 & 0 & 1 & 1 & 0 & 0 & 1 & 0 & 1 & 0 \\
\hline PEMB89 & 0 & 0 & 0 & 1 & 0 & 1 & 0 & 1 & 0 & 0 \\
\hline PEMB91 & 1 & 1 & 1 & 1 & 0 & 0 & 0 & 1 & 0 & 0 \\
\hline PEMB92 & 1 & 1 & 1 & 1 & 1 & 0 & 0 & 1 & 1 & 0 \\
\hline PEMB95 & 0 & 0 & 1 & 1 & 0 & 0 & 0 & 0 & 1 & 0 \\
\hline PEMB97 & 1 & 1 & 1 & 1 & 0 & 0 & 0 & 1 & 1 & 0 \\
\hline PEMB98 & 1 & 1 & 1 & 1 & 0 & 0 & 0 & 1 & 1 & 0 \\
\hline РEMB99 & 1 & 0 & 1 & 0 & 0 & 0 & 0 & 1 & 0 & 0 \\
\hline Total Items Sold & 26 & 27 & 28 & 28 & 9 & 12 & 27 & 27 & 27 & 12 \\
\hline
\end{tabular}


1) Market Basket Analysis Phase Using Apriori Algorithms.

a) Analysis of high frequency patterns

High frequency pattern analysis is done by finding a combination of items that meet the minimum requirements of the support value in the transaction data. The data in Table 3 shows the results of the number of transactions in each itemset:

Table 3. List of itemset.

\begin{tabular}{|c|c|c|c|c|c|}
\hline Numb & Code BR & Name BR & $\begin{array}{c}\text { Total } \\
\text { Transactions }\end{array}$ & $\begin{array}{c}\text { Initial } \\
\text { Inventory }\end{array}$ & $\begin{array}{c}\text { Stock } \\
\text { Available }\end{array}$ \\
\hline 1 & 0000001 & $\begin{array}{l}\text { Black } \\
\text { Coffee 1/2 } \\
\text { Kg }\end{array}$ & 26 & 45 & 19 \\
\hline 2 & 0000002 & $\begin{array}{l}\text { Granulated } \\
\text { Sugar } 1 \mathrm{Kg}\end{array}$ & 27 & 30 & 3 \\
\hline 3 & 0000003 & $\begin{array}{l}\text { Granulated } \\
\text { Sugar } 1 / 2 \\
\mathrm{Kg}\end{array}$ & 28 & 40 & 12 \\
\hline 4 & ASL101 & $\begin{array}{l}\text { Gallon } \\
\text { Bottled } \\
\text { Water }\end{array}$ & 28 & 80 & 52 \\
\hline 5 & ASL102 & $\begin{array}{l}600 \mathrm{ml} \\
\text { Bottled } \\
\text { Water }\end{array}$ & 9 & 240 & 231 \\
\hline 6 & ASL103 & $\begin{array}{l}\text { Glass } \\
\text { drinking } \\
\text { water }\end{array}$ & 12 & 40 & 28 \\
\hline 7 & BR1111 & $\begin{array}{l}\text { Cheap rice } \\
5 \mathrm{~kg}\end{array}$ & 27 & 40 & 13 \\
\hline 8 & BR1112 & $\begin{array}{l}\text { Premium } \\
\text { Rice } 5 \mathrm{Kg}\end{array}$ & 27 & 40 & 13 \\
\hline 9 & GL1001 & $\begin{array}{l}\text { Javanese } \\
\text { Sugar } 1 \mathrm{Kg}\end{array}$ & 27 & 40 & 13 \\
\hline 10 & GL1002 & $\begin{array}{l}\text { Java Sugar } \\
1 / 2 \mathrm{Kg}\end{array}$ & 12 & 60 & 48 \\
\hline
\end{tabular}

The next step is to calculate the value of support in combination with 1 - itemset or C_1 using equation (1):

$$
\text { Support }(A)=\frac{\text { Number of Transactions Containing } A}{\text { Transaction Total }}
$$

The results of determining the support value of each item can be seen in table 4 :

Table 4. Results for 1-itemset support values

\begin{tabular}{cclcc}
\hline NUMB & $\begin{array}{c}\text { Code } \\
\text { BR }\end{array}$ & Name BR & $\begin{array}{c}\text { Total } \\
\text { Transactions }\end{array}$ & $\begin{array}{c}\text { Support } \\
(\mathbf{\%})\end{array}$ \\
\hline $\mathbf{1}$ & 0000001 & $\begin{array}{l}\text { Black Coffee } \\
1 / 2 \mathrm{Kg}\end{array}$ & 26 & $\mathbf{5 2}$ \\
$\mathbf{2}$ & 0000002 & $\begin{array}{l}\text { Granulated } \\
\text { Sugar 1 Kg }\end{array}$ & 27 & $\mathbf{5 4}$ \\
$\mathbf{3}$ & 0000003 & $\begin{array}{l}\text { Granulated } \\
\text { Sugar 1/2 } \\
\mathrm{Kg}\end{array}$ & 28 & $\mathbf{5 6}$ \\
\hline
\end{tabular}

\begin{tabular}{cclcc}
\hline NUMB & $\begin{array}{c}\text { Code } \\
\text { BR }\end{array}$ & Name BR & $\begin{array}{c}\text { Total } \\
\text { Transactions }\end{array}$ & $\begin{array}{c}\text { Support } \\
(\mathbf{\%})\end{array}$ \\
\hline $\mathbf{4}$ & ASL101 & $\begin{array}{l}\text { Gallon } \\
\text { Bottled } \\
\text { Water } \\
600 \mathrm{ml}\end{array}$ & 28 & $\mathbf{5 6}$ \\
$\mathbf{5}$ & ASL102 & $\begin{array}{l}\text { Bottled } \\
\text { Water } \\
\text { Glass } \\
\text { drinking } \\
\text { water }\end{array}$ & 12 & $\mathbf{1 8}$ \\
$\mathbf{6}$ & ASL103 & $\mathbf{2 4}$ \\
$\mathbf{7}$ & BR1111 & $\begin{array}{l}\text { Cheap rice } \\
5 \text { kg }\end{array}$ & 27 & $\mathbf{5 4}$ \\
$\mathbf{8}$ & BR1112 & $\begin{array}{l}\text { Premium } \\
\text { Rice 5 Kg }\end{array}$ & 27 & $\mathbf{5 4}$ \\
$\mathbf{9}$ & GL1001 & $\begin{array}{l}\text { Javanese } \\
\text { Sugar 1 Kg }\end{array}$ & 27 & $\mathbf{5 4}$ \\
$\mathbf{1 0}$ & GL1002 & $\begin{array}{l}\text { Java Sugar } \\
1 / 2 \text { Kg }\end{array}$ & 12 & $\mathbf{2 4}$ \\
\hline
\end{tabular}

The next step is to identify a combination pattern with a minimum support of $50 \%$, then the process of forming 2 - itemset or C_2 with a minimum support of $50 \%$ as shown in table 5 .

Table 5. Combination patterns of $\mathrm{C} \_1$ with a minimum support of $50 \%$.

\begin{tabular}{|c|c|c|c|c|}
\hline Numb & $\begin{array}{c}\text { Code } \\
\text { BR }\end{array}$ & Name BR & Total Transactions & Support (\%) \\
\hline 1 & 0000001 & $\begin{array}{l}\text { Black } \\
\text { Coffee 1/2 } \\
\text { Kg }\end{array}$ & 26 & 52 \\
\hline 2 & 0000002 & $\begin{array}{l}\text { Granulated } \\
\text { Sugar } 1 \mathrm{Kg}\end{array}$ & 27 & 54 \\
\hline 3 & 0000003 & $\begin{array}{l}\text { Granulated } \\
\text { Sugar } 1 / 2 \\
\mathrm{Kg}\end{array}$ & 28 & 56 \\
\hline 4 & ASL101 & $\begin{array}{l}\text { Gallon } \\
\text { Bottled } \\
\text { Water }\end{array}$ & 28 & 56 \\
\hline 5 & BR1111 & $\begin{array}{l}\text { Cheap rice } \\
5 \mathrm{~kg}\end{array}$ & 27 & 54 \\
\hline 6 & BR1112 & $\begin{array}{l}\text { Premium } \\
\text { Rice } 5 \mathrm{Kg}\end{array}$ & 27 & 54 \\
\hline 7 & GL1001 & $\begin{array}{l}\text { Javanese } \\
\text { Sugar } 1 \mathrm{Kg}\end{array}$ & 27 & 54 \\
\hline
\end{tabular}

Calculation of support values using a 2-itemset combination using equation (2):

Support $(A, B)=P(A \cap B)$

$\operatorname{Support}(A, B)=\frac{\sum \text { transaction contains } A \text { and } B}{\Sigma \text { Total } \text { Transactions }}$ 
Table 6. Minimum support values of $50 \%$ of the 2 -itemset combination

\begin{tabular}{clcc}
\hline Numb & \multicolumn{1}{c}{ Item Name } & Amount & $\begin{array}{c}\text { Support } \\
(\%)\end{array}$ \\
\hline 1 & Black Coffee $1 / 2 \mathrm{Kg} \rightarrow$ Granulated Sugar 1 Kg & 19 & 38 \\
2 & Black Coffee $1 / 2 \mathrm{Kg} \rightarrow$ Granulated Sugar 1/2 Kg & 18 & 36 \\
3 & Black Coffee $1 / 2 \mathrm{Kg} \rightarrow$ Gallon Bottled Drinking Water & 5 & 10 \\
4 & Black Coffee $1 / 2 \mathrm{Kg} \rightarrow$ Cheap Rice 5 Kg & 12 & 24 \\
5 & Black Coffee 1/2 Kg $\rightarrow$ Premium Rice 5 Kg & 16 & 32 \\
6 & Black Coffee 1/2 Kg $\rightarrow$ Java Sugar 1 Kg & 14 & 28 \\
7 & Granulated Sugar 1 Kg $\rightarrow$ Granulated Sugar 1/2 Kg & 16 & 32 \\
8 & Granulated Sugar 1 Kg $\rightarrow$ Gallon Bottled Drinking Water & 13 & 26 \\
9 & Granulated Sugar 1 Kg $\rightarrow$ Cheap Rice 5 Kg & 12 & 24 \\
10 & Granulated Sugar 1 kg $\rightarrow$ Premium Rice 5 kg & 13 & 26 \\
11 & Granulated Sugar 1 kg $\rightarrow$ Javanese Sugar 1 kg & 13 & 26 \\
12 & Gallon Bottled Drinking Water $\rightarrow$ Cheap Rice 5 Kg & 13 & 26 \\
13 & Gallon Bottled Drinking Water $\rightarrow$ Premium Rice 5 Kg & 14 & 28 \\
14 & Gallon Bottled Drinking Water $\rightarrow$ Java Sugar 1 Kg & 18 & 36 \\
15 & Cheap Rice 5 Kg $\rightarrow$ Premium Rice 5 Kg & 13 & 26 \\
16 & Cheap Rice 5 Kg $\rightarrow$ Java Sugar 1 Kg & 15 & 30 \\
17 & Premium Rice 5 Kg $\rightarrow$ Java Sugar 1 Kg & 12 & 24 \\
\hline
\end{tabular}

In the process of forming a 2-itemset combination pattern no one meets the minimum support value of $50 \%$, then a 1 -itemset combination can be met for the formation of associative rules.

2) Establishment of associative rules

After a minimum support value of $50 \%$ of the C_1 combination is determined, an associative rule is sought that meets the minimum confidence requirement of candidate 2 item A containing B in each itemset. The confidence value of the associative rule 2 - item $\mathrm{A} \rightarrow \mathrm{B}$ is obtained using equation (3):

Confidence $(\mathrm{A}, \mathrm{B})=$

$P(B \mid A)=\frac{\text { Number of transactions containing } A \text { and } B}{\text { Number of transactions containing } A}$

Table 7. Confidence values from forming 2 -item associative rules $\mathrm{A} \rightarrow \mathrm{B}$.

\begin{tabular}{|c|c|c|c|}
\hline Numb & Itemset & Amount & Confident $(\%)$ \\
\hline 1 & (0000001, Black Coffee 1/2 Kg), (0000002, 1 Kg Granulated Sugar) & 18 & 69,23 \\
\hline 2 & (0000001, Black Coffee 1/2 Kg), (0000003, Granulated Sugar 1/2 Kg) & 18 & 69,23 \\
\hline 3 & (0000001, Black Coffee 1/2 Kg), (ASL101, Gallon Bottled Drinking Water) & 15 & 57,69 \\
\hline 4 & (0000001, Black Coffee 1/2 Kg), (BR1111, Cheap Rice 5 Kg) & 13 & 50,00 \\
\hline 5 & (0000001, Black Coffee 1/2 Kg), (BR1112, Premium Rice 5 Kg) & 16 & 61,53 \\
\hline 6 & (0000001, Black Coffee 1/2 Kg), (GL1001, Javanese Sugar 1 Kg) & 14 & 53,84 \\
\hline 7 & (0000002, 1 Kg Granulated Sugar), (0000003, 1/2 Kg Granulated Sugar) & 16 & 59,25 \\
\hline 8 & $\begin{array}{l}\text { (0000002, } 1 \text { Kg Granulated Sugar), (ASL101, Gallon Bottled Drinking } \\
\text { Water) }\end{array}$ & 13 & 48,14 \\
\hline 9 & (0000002, 1 Kg Granulated Sugar), (BR1111, Cheap Rice 5 Kg) & 12 & 44,44 \\
\hline 10 & (0000002, 1 Kg Granulated Sugar), (BR1112, Premium Rice 5 Kg) & 13 & 48,18 \\
\hline 11 & (0000002, 1 Kg Granulated Sugar), (GL1001, 1 Kg Java Sugar) & 13 & 48,14 \\
\hline 12 & $\begin{array}{l}\text { (0000003, Granulated Sugar 1/2 Kg), (ASL101, Gallon Bottled Drinking } \\
\text { Water) }\end{array}$ & 20 & 71,42 \\
\hline
\end{tabular}




\begin{tabular}{clcc}
\hline Numb & \multicolumn{1}{c}{ Itemset } & Amount & Confident (\%) \\
\hline 13 & (0000003, Granulated Sugar 1/2 Kg), (BR1111, Cheap Rice 5 Kg) & 16 & 57,14 \\
14 & (0000003, Granulated Sugar 1/2 Kg), (BR1112, Premium Rice 5 Kg) & 12 & 42,85 \\
15 & (0000003, Granulated Sugar 1/2 Kg), (GL1001, Java Sugar 1 Kg) & 18 & 64,28 \\
16 & (ASL101, Gallon Bottled Water), (ASL102, 600ml Bottled Water) & 6 & 21,42 \\
17 & (ASL101, Gallon Bottled Drinking Water), (BR1111, Cheap Rice 5 Kg) & 13 & 46,42 \\
18 & (ASL101, Gallon Bottled Drinking Water), (BR1112, Premium Rice 5 Kg) & 14 & 50,00 \\
19 & (ASL101, Gallon Bottled Drinking Water), (GL1001, Java Sugar 1 Kg) & 18 & 64,28 \\
20 & (BR1111, Cheap Rice 5 Kg), (BR1112, Premium Rice 5 Kg) & 13 & 48,14 \\
21 & (BR1111, Cheap Rice 5 Kg), (GL1001, Java Sugar 1 Kg) & 14 & 51,85 \\
22 & (BR1112, Premium Rice 5 Kg), (GL1001, Java Sugar 1 Kg) & 14 & 51,85 \\
\hline
\end{tabular}

Next is determining the minimum confidence value of $60 \%$, the confidence value of 2 - items $\mathrm{A} \rightarrow \mathrm{B}$ that will qualify for the determination of association rules has a confidence value above $60 \%$.

Table 8. Value of Association rules with a minimum value of $60 \%$ confidence.

\begin{tabular}{clcc}
\hline Numb & \multicolumn{1}{c}{ Itemset } & Amount & Confident (\%) \\
\hline 1 & $(0000001$, Black Coffee $1 / 2 \mathrm{Kg}) \rightarrow(0000002,1 \mathrm{Kg}$ Granulated Sugar $)$ & 18 & 69,23 \\
2 & $(0000001$, Black Coffee $1 / 2 \mathrm{Kg}) \rightarrow(0000003$, Granulated Sugar 1/2 Kg) & 18 & 69,23 \\
3 & $(0000001$, Black Coffee $1 / 2 \mathrm{Kg}) \rightarrow(\mathrm{BR} 1112$, Premium Rice $5 \mathrm{Kg})$ & 16 & 61,53 \\
4 & $(0000003$, Granulated Sugar $1 / 2 \mathrm{Kg}) \rightarrow($ ASL101, Gallon Bottled Drinking Water $)$ & 20 & 71,42 \\
5 & $(0000003$, Granulated Sugar $1 / 2 \mathrm{Kg}) \rightarrow($ GL1001, Java Sugar 1 Kg $)$ & 18 & 64,28 \\
6 & $($ ASL101, Gallon Bottled Drinking Water $) \rightarrow($ GL1001, Java Sugar 1 Kg) & 18 & 64,28 \\
\hline
\end{tabular}

Table 9. Results of determining the pattern of handling stock of goods.

\begin{tabular}{cclcccc}
\hline NUMB & KODE BR & \multicolumn{1}{c}{ NAME BR } & $\begin{array}{c}\text { TOTAL } \\
\text { TRANSACTIONS }\end{array}$ & $\begin{array}{c}\text { SUPPORT } \\
(\%)\end{array}$ & $\begin{array}{c}\text { INITIAL } \\
\text { INVENT- } \\
\text { ORY }\end{array}$ & $\begin{array}{c}\text { ADDITI-ON } \\
\text { STOCK }\end{array}$ \\
\hline 1 & 0000001 & Black Coffee 1/2 Kg & 26 & 52 & 45 & 23 \\
2 & 0000002 & Granulated Sugar 1 Kg & 27 & 54 & 30 & 16 \\
3 & 0000003 & Granulated Sugar 1/2 Kg & 28 & 56 & 40 & 22 \\
4 & ASL101 & Gallon Bottled Water & 28 & 56 & 52 & 29 \\
5 & BR1111 & Cheap rice 5 kg & 27 & 54 & 40 & 22 \\
6 & BR1112 & Premium Rice 5 Kg & 27 & 54 & 40 & 22 \\
7 & GL1001 & Javanese Sugar 1 Kg & 27 & 54 & 40 & 22 \\
\hline
\end{tabular}

Table 10. Association Rules

\begin{tabular}{clc}
\hline Numb & \multicolumn{1}{c}{ Rules } & Confidence \% \\
\hline 1 & If you buy $1 / 2 \mathrm{Kg}$ Black Coffee then buy 1 Kg Granulated Sugar & 69,23 \\
2 & If you buy $1 / 2 \mathrm{Kg}$ Black Coffee then buy $1 / 2 \mathrm{Kg}$ Sugar & 69,23 \\
3 & If you buy $1 / 2 \mathrm{Kg}$ Black Coffee then buy 5 Kg Premium Rice & 61,53 \\
4 & If you buy 1/2 Kg Granulated Sugar then buy Gallon Bottled Drinking & 71,42 \\
5 & Water & If you buy 1/2 Kg Granulated Sugar then buy 1 kg of Java Sugar \\
6 & If you buy Gallon Bottled Drinking Water then buy Java Sugar 1 Kg & 64,28 \\
\hline
\end{tabular}




\section{c. Results Determination Stage}

From the results of the analysis of determining the value of support and confidence, it can be concluded the results of the pattern of handling stock of goods and arrangement of goods on the store shelf, as follows:

1. Pattern of handling stock of goods

From the results of determining the minimum support value of $50 \%$ in the 1 -itemset combination pattern, it can be identified the addition of stock items with a percentage:

Stock Addition $=\frac{\text { Initial inventory }}{\text { Value of Support }(\%)} \times 100 \%$

From Table 9 results can be drawn from the stage of identifying the pattern of handling stock of goods utilizing a minimum value of support of $50 \%$ of the combination of 1-itemset, by calculating the initial inventory is compared with the value of support so that it can be identified in the item stock increase column in Table 9.

2. Pattern of Arrangement of Goods

From the results of the analysis in identifying the handling of the layout of the goods using Apriori Algorithm, it can be determined from the minimum value of $50 \%$ support and the minimum value of Confidence $60 \%$ which produces the following association rules:

From Table 10. it can be identified the tendency of customer purchases, it is known that the tendency of customers if buying Black Coffee items $1 / 2 \mathrm{Kg}$ is most likely with a Confidence value of $69.23 \%$ customers will also buy $1 \mathrm{Kg}$ Granulated Sugar. from this tendency can be a strategy to arrange the layout of the item with the pattern of Black Coffee $1 / 2$ $\mathrm{Kg}$ brought closer to $1 \mathrm{Kg}$ Granulated Sugar, Black Coffee $1 / 2 \mathrm{Kg}$ closer to Granulated Sugar $1 / 2 \mathrm{Kg}$ and so on, so that customers can easily find the items they need.

\section{Conclusion}

Based on the analysis phase of the results and discussion above, it can be concluded that identification of patterns of handling stock of goods and arrangement of goods can utilize data on the results of sales transactions. And then, analysis of high frequency patterns with a minimum support value of $50 \%$ from a combination of 1 - itemset $\mathrm{C} 1$ can determine the pattern of handling stock of goods, namely by balancing the initial inventory with a support value so that the prediction results of adding stock will be obtained. Beside that, the results of the formation of association rules that are determined from a minimum value of $50 \%$ support and a minimum value of $60 \%$ confidence can produce a tendency of customers to buy items, so that these tendencies can be a reference in the process of item layout by arranging items close together.

\section{References}

[1] A. Junaidi, "Implementasi Algoritma Apriori dan FP-Growth untuk Menentukan Persediaan Barang," Jurnal Sisfokom (Sistem Informasi dan Komputer), vol. 8, no. 1, pp. 61-67, Maret 2019.

[2] H. Jiawei dan K. Micheline, Data Mining: Concept and Techniques Second Edition, Morgan Kaufman Publishers, 2006.

[3] D. T. Larose, Discovering knowledge in data : an introduction data mining, Jhon Wiley \& Sons Inc, 2005.

[4] Suprayogi, dan A. Karima, "Implemetasi Algoritma Apriori dengan Market Basket Analysis untuk Pengaturan Tata Letak Produk," Jurnal SISFOTENIKA, vol. 9, no. 2, pp. 169-179, Juli 2019.

[5] Maharani, N. A. Hasibuan, dan N. Silalahi, "Implementasi Data Mining untuk Pengaturan Layout Minimarket dengan Menerapkan Association Rule," Jurnal Riset Komputer, vol. 4, no. 4, pp. 6-11, Agustus 2017.

[6] A. K. Prasidya, dan C. Fibriani "Analisis Kaidah Asosiasi Antar Item Dalam Transaksi Pembelian Menggunakan Data Mining Dengan Algoritma Apriori (Studi Kasus Gun Bandungan Jawa Tengah," Jurnal Ilmiah Teknologi Informasi, vol. 14, no. 2, pp. 173-184, Juli 2017.

[7] M. Sholik, dan A. Salam, "Implementasi Algoritma Apriori untuk Mencari Asosiasi BArang yang Dijual di E-commerce OrderMAs," Jurnal Techno. Com, vol. 17, no. 2, pp. 158-170, Mei 2018.

[8] G. Abdurrahman, "Analisis Aturan Asosiasi Data Transaksi Supermarket Menggunakan Algoritma Apriori," Jurnal Sistem dan Teknologi Informasi Indonesia, vol. 2, no.2, pp. 100-111, Agustus 2017.

[9] R. Yanto, dan R. Khorirah, "Implementasi Data Mining dengan Metode Algoritma Apriori dalam Menentukan Pola Pembelian Obat," Creative Information Technology Journal, vol. 2, no. 2, pp. 102-113, April 2015.

[10] S. Wahyuni, Suherman, dan L. P. Harahap, "Implementasi Data Mining dalam Memprediksi Stok Barang Menggunakan Algoritma Apriori," Jurnal Teknik dan Informatika, vol. 5, no.2, pp. 67-71, Juli 2018.

[11] E. D. Sikumbang, "Penerapan Data Mining Penjualan Sepatu Menggunakan Metode Algoritma Apriori," Jurnal Teknik Komputer, vol. 4, no. 1, pp. 156-161, Februari 2018.

[12] Y. Suardi, B. F. Ahmad, M. Ita, dan W. Bambang, 
"Penerapan Data Mining Pengaturan Pola Tata Letak Barang pada Berkah Swalayan untuk Strategi Penjualan Menggunakan Algoritma Apriori," Jurnal Teknologi Sistem Informasi dan Sistem Komputer TGD, vol. 2, no. 1, pp. 69-75, Januari 2019.

[13] A. Cep, H. Nila, dan W. Wiwiek, "Implementasi Data Mining Penjualan Kosmetik Pada Toko Zahrani Menggunakan Algoritma Apriori," Sentra Penelitian Engineering dan Edukasi, vol. 11, no. 2, pp. 1-7, Mei 2019.

[14] B. A. Pilipus, R. J. Ekik, dan L. Yonata, "Prediksi Pola Pembelian Customer dengan Market Basket Analysis pada PT. Capella Medan," Jurnal Sistem Informasi dan Komputer, vol. 2, no. 2, pp. 59-66, Maret 2019.

[15] Y. S. Haysrif, Rismayani, dan L. S Novita, "Data Mining Menggunakan Algoritma Apriori untuk Analisis Penjualan," Seminar Ilmiah Sistem Informasi Dan Teknologi Informasi, vol. 6, no. 1, pp. 217-226, Agustus 2019. 